\title{
Generating dithering noise for maximum likelihood estimation from quantized data
}

\author{
Fredrik Gustafsson and Rickard Karlsson
}

\section{Linköping University Post Print}

N.B.: When citing this work, cite the original article.

Original Publication:

Fredrik Gustafsson and Rickard Karlsson, Generating dithering noise for maximum likelihood estimation from quantized data, 2013, Automatica, (49), 2, 554-560.

http://dx.doi.org/10.1016/j.automatica.2012.11.028

Copyright: Elsevier

http://www.elsevier.com/

Postprint available at: Linköping University Electronic Press

http://urn.kb.se/resolve?urn=urn:nbn:se:liu:diva-90218 


\title{
Generating Dithering Noise for Maximum Likelihood Estimation from Quantized Data
}

\author{
Fredrik Gustafsson ${ }^{1}$ Rickard Karlsson ${ }^{2}$
}

\begin{abstract}
The Quantization Theorem I (QT I) implies that the likelihood function can be reconstructed from quantized sensor observations, given that appropriate dithering noise is added before quantization. We present constructive algorithms to generate such dithering noise. The application to maximum likelihood estimation (MLE) is studied in particular. In short, dithering has the same role for amplitude quantization as an anti-alias filter has for sampling, in that it enables perfect reconstruction of the dithered but unquantized signal's likelihood function. Without dithering, the likelihood function suffers from a kind of aliasing expressed as a counterpart to Poisson's summation formula which makes the exact MLE intractable to compute. With dithering, it is demonstrated that standard MLE algorithms can be re-used on a smoothed likelihood function of the original signal, and statistically efficiency is obtained. The implication of dithering to the Cramér-Rao Lower Bound (CRLB) is studied, and illustrative examples are provided.
\end{abstract}

\section{Introduction}

Quantization was a well studied topic some decades ago, [15], when the underlying reason was the finite precision in electronics and micro-processors. Today, new reasons have appeared that motivate a revisit of the area. One is that cheap low-quality sensors have appeared on the market which opens up many new application areas for embedded algorithms, where the sensor resolution is much worse than the micro-processor resolution. Some sensors are naturally quantized as radar range, vision (pixel quantization), cogged wheels to measure angular speeds etc. Sensor networks is one hot research topic where this work fits in. The conclusion is that quite advanced pre-processing at the sensor node is possible to mitigate the effects of sensor quantization.

This contribution regards the sensor readings as the only instance where quantization effects are important. All sub-sequent computations are done with floating point precision, or in fixed-point arithmetics with adaptive scaling of all numbers, which means that internal quantization effects can be neglected. As one example in this direction, [2] shows that dithering helps a network to reach consensus for estimating a signal mean when quantized samples from different nodes are communicated. The paper [10] motivates the parameter estimation problem

\footnotetext{
* This work was supported by the Swedish Research Council through the center of excellence CADICS and project grant "fundamental issues in sensor fusion".

1 Dept. of Elec. Eng., Linköping University, Sweden. E-mail: fredrik@isy.liu.se

2 Nira Dynamics AB, Linköping, Sweden. E-mail: rickard.karlsson@niradynamics.se
}

in sensor network further, and develops some feedback strategies to the sensors in the same spirit as in [1]. Statistical treatment of quantization effects was developed in [21, 23], and the newer statistical analysis as surveyed in $[20,22]$. They show that quantization adds two kind of errors to the measurement, the first one is a direct effect that can be modeled as additive uniform noise (AUN), and the other one is an intrinsic alias like uncertainty, where fast variations in the probability density function (PDF) of the measurement noise are folded to low "frequencies" in the CF domain (see Section 3.2). It is also known, [20], that adding $r$ uniformly distributed samples as dithering noise enables reconstruction of all the first $r$ moments of the unquantized signal, when uniform quantization is considered, where applications to system identification is studied in $[7,8]$. This is known as Quantization Theorem II (QT II). However, reconstruction of moments is easier than reconstruction of the complete amplitude distribution and in particular the likelihood function. The aliasing in the amplitude distribution can be avoided by adding proper dithering noise, and this enables that the likelihood function can be reconstructed from quantized sensor observations (QT I). Since this noise plays the same role in quantization and PDF reconstruction as an anti-alias filter does for sampling and reconstruction, such dithering noise will be referred to as band-limited (BL) noise. The theoretical requirements for a BL noise were given in the fifties in [21] and later revisited in [18].

A first contribution is to describe constructive ways to generate such noise. Two methods are provided for generating BL dithering noise, and one concrete algorithm based on accept-reject sampling. For most cases adding 
dithering noise inevitably destroys information. This is in perfect analogy with lowpass filtering used to avoid frequency aliasing. Information is thus lost, but it is at least not mis-interpreted leading to an estimation bias. For the case of parameter estimation in 1-bit quantized samples, [5, 6] design the dithering noise by MSE optimization, trading off a bias decrease to the variance increase due to dithering. Adding a suitably designed dithering noise should simplify the derivation of estimators. A second contribution is to utilize this new class of dithering noise to maximum likelihood estimation problems. The dithering noise implies that the discrete PDF of the quantized signal can be computed by convolving the original likelihood with first the dithering noise PDF and then the PDF of a uniform distribution. That is, quantization can under some conditions be regarded as adding uniformly distributed noise without approximation regarding moment calculations and the likelihood can be reconstructed by lowpass filtering the discrete PDF.

The paper is organized as follows: Section 2 formalizes the problem definition and provides some simple motivating examples. Section 3 summarizes the most important concepts from statistical quantization theory needed for the derivations. Section 4 presents the new methods to generate band-limited noise. In Section 5, ML-estimation for different quantization cases are presented, and a performance bound is calculated. Section 6 concludes the paper.

\section{Motivation and Problem Formulation}

\subsection{Signal Model}

The signal model in this contribution includes a dithering noise $d_{k}$ that is added to a stochastic signal $z_{k}$ before it is quantized to $q_{k}$,

$$
\begin{aligned}
& y_{k}=z_{k}+d_{k}, \\
& q_{k}=\mathcal{Q}_{m}\left(y_{k}\right) .
\end{aligned}
$$

Here, $\mathcal{Q}_{m}(\cdot)$ denotes the quantization operator

$$
\mathcal{Q}_{m}\left(y_{k}\right)= \begin{cases}-m \Delta+\frac{\Delta}{2}, & y_{k}<-(m-1) \Delta \\ \Delta\left\lfloor\frac{y_{k}}{\Delta}\right\rfloor+\frac{\Delta}{2}, & -(m-1) \Delta \leq y_{k}<(m-1) \Delta \\ m \Delta-\frac{\Delta}{2}, & y_{k} \geq(m-1) \Delta\end{cases}
$$

where the floor operator $\lfloor x\rfloor$ denotes the largest integer that is smaller than or equal to $x, \mathcal{Q}_{1}(y)$ defines the binary quantizer and where $\mathcal{Q}_{\infty}(y)$ is defined as the unsaturated quantization function.

\subsection{Maximum Likelihood Estimation}

Consider now an estimation problem, where the signal $z_{k}(\theta), k=1,2, \ldots, N$ depends on a vector $\theta$ of unknown parameters and the problem is to make inference of $\theta$ from the quantized but possibly dithered observations

$$
q_{k}=\mathcal{Q}_{m}\left(z_{k}(\theta)+d_{k}\right), \quad k=1,2, \ldots, N .
$$

Without quantization, there are many standard methods for solving the maximum likelihood (ML) method, where the expectation maximization (EM) algorithm is possibly the most common one. For quantization two approaches will be discussed in the sequel: applying the ML method directly or using dithering to simplify the problem.

\subsubsection{General MLE for Quantization}

Parameter estimation using the ML method for quantization has been discussed in for instance [3, 8, 11]. A simple but instructive example is the unknown signal mean model $z_{k}(\theta)=x+e_{k}$, where $e_{k}$ denotes white measurement noise and the parameter vector $\theta=\left(x, \sigma_{e}^{2}\right)$ contains the mean $x$ and possible also the variance $\sigma_{e}^{2}=$ $\mathbb{E}\left(e_{k}^{2}\right)$. This example can be extended to linear models $z_{k}=H x+e_{k}$, nonlinear model $z_{k}=h(x)+e_{k}$, nonlinear filtering $z_{k}=h\left(x_{k}\right)+e_{k}$ (where the state $x_{k}$ varies over time according to a dynamic model) and system identification, where $\theta$ contains parameters in a dynamic model. The ML method is applicable to all these cases, where the ML estimate (MLE) is defined as

$$
\hat{\theta}^{M L}=\arg \max _{\theta} \begin{cases}\prod_{k=1}^{N} p_{z \mid \theta}\left(z_{k}\right), & \text { if un-quantized }, \\ \prod_{k=1}^{N} p_{q \mid \theta}\left(q_{k}\right), & \text { if quantized. }\end{cases}
$$

The MLE is statistically efficient, defined so that the bias as well as the variance tend to zero as the number of samples $N$ increases. Computing the MLE using the likelihood $p_{q \mid \theta}\left(q_{k}\right)$ directly is mathematically intractable in most cases and also suffers from the curse of dimensionality. Example 1 illustrates one principal problem.

Example 1 Consider the problem of estimating $\theta$ in the Gaussian distribution $z_{k}(\theta) \sim \mathcal{N}\left(\theta, \theta^{2}\right)$. The MLE can be shown to be

$$
\hat{\theta}^{M L}=-\frac{\bar{z}}{2}+\sqrt{\frac{\bar{z}^{2}}{4}+\overline{z^{2}}}
$$

where $\bar{z}=\frac{1}{N} \sum_{k=1}^{N} z_{k}$ and $\overline{z^{2}}=\frac{1}{N} \sum_{k=1}^{N} z_{k}^{2}$. For quantized observations $q_{k}(\theta)=\mathcal{Q}_{\infty}\left(z_{k}(\theta)\right)$ and $\Delta=1$, each observation is mapped to an integer $i$, and the likelihood for each quantized observation $q_{k}=i \Delta+\Delta / 2$ is given by

$$
p_{i \mid \theta}\left(q_{k}\right)=\int_{i \Delta}^{(i+1) \Delta} \frac{1}{\sqrt{2 \pi} \theta} e^{-\frac{\left(q_{k}-\theta-z\right)^{2}}{2 \theta^{2}}} d z .
$$

The likelihood function can be differentiated in $\theta$, however in general it can be quite a lot of work doing so and an exhaustive search over the integer space $i=0, \pm 1, \pm 2, \ldots$. is required to compute the MLE. 

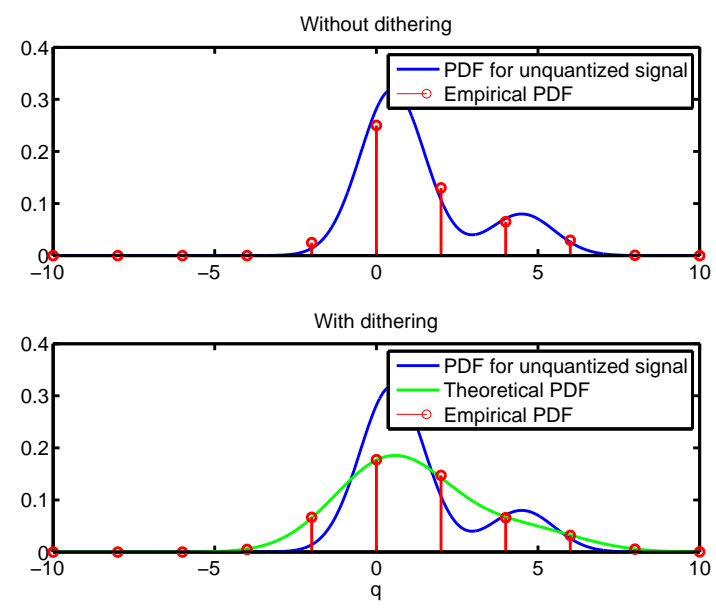

Fig. 1. Quantization of stochastic variables from a Gaussian mixture. The first subplot relates the empirical histogram of quantized observations versus the original (unquantized) likelihood function $p_{y \mid \theta}(y)$. The second subplot shows the theoretical likelihood function (6) of the quantized data after dithering $p_{q_{k} \mid \theta}\left(q_{k}\right)$ together with the empirical distribution.

\subsubsection{MLE using Dithering}

As indicated in (2) dithering can simplify estimation when dealing with quantized signals. In Section 4 a special class of dithering noise that allows perfect reconstruction of the dithered but unquantized signal is introduced, so the MLE can be computed using the following likelihood

$$
p_{q \mid \theta}\left(q_{k}\right)=p_{z \mid \theta} \star p_{d} \star p_{u}\left(q_{k}\right),
$$

where $\star$ denotes convolution, $p_{d}$ is the PDF of the dithering noise, and $p_{u}$ denotes the PDF of a uniform distribution. That is, the function to be minimized is the original likelihood function in (3), smoothed with the PDF of dithering noise and a uniform distribution. Example 2 illustrates this.

Example 2 Consider the following Gaussian mixture

$$
z_{k}(\theta) \sim \alpha \mathcal{N}\left(\mu_{1}, P_{1}\right)+(1-\alpha) \mathcal{N}\left(\mu_{2}, P_{2}\right)
$$

with parameters $\theta=\left(\alpha, \mu_{1}, \mu_{2}, P_{1}, P_{2}\right)^{T}$, and true values $\theta^{\circ}=(0.8,0.5,4.5,1,1)^{T}$. Such mixture models are appropriate for many distance measuring devices where multi path causes a positive mode of the noise distribution.

The first subplot in Fig. 1 relates the empirical histogram of quantized observations $(\Delta=1)$ versus the original (unquantized) likelihood function $p_{y \mid \theta}(y)$. One can here notice a small but significant difference. Parameter estimation based on the likelihood function for unquantized observations would lead to a bias. The second subplot shows the theoretical likelihood function of the quantized data after dithering $p_{q \mid \theta}(q)$. Now, the fit of empirical data to the theoretical distribution is perfect. Further, $p_{q \mid \theta}(q)$ is a smooth function of the parameters $\theta$, enabling efficient gradient search algorithms.

\subsection{MLE Summary}

To summarize, quantization can be approximated with independent uniform noise, but this is in many cases a coarse approximation. From the MLE point-of-view with BL dithering noise, quantization can be interpreted as adding dithering noise and uniform noise to the output, and this is no approximation. The price for this is an increased variance of the additive dithering noise, which will be discussed later in terms of the CRLB. Another approach is to apply the ML method directly, which theoretically would be the best choice. However, due to the intractable computational burden for instance due to exhaustive search in the parameter space this might not be feasible in general.

\section{Statistical Quantization Theory}

This section will review basic quantization theory as presented in for instance [22]. In this context, the quantized measurement $q$ contains the following uncertainties:

- The measurement noise, which is the same as for the unquantized signal $y$.

- The first order effect of the quantization level $\Delta$ which will be referred to as the additive uniform noise (AUN) effect, with the corresponding variance $\Delta^{2} / 12$.

- Remaining high order effects, are interpreted as PDF aliasing (analogy with sampling theory).

- Saturation effects when $|y|>m \Delta$.

Parameter dependence and saturation will be ignored for the rest of this section, where the goal is to analyze the alias effect.

\subsection{Probability Density Function After Quantization}

The nice exposition of quantization seen as area sampling from [23] is reviewed here. Define the probability function as

$$
p_{i}=\operatorname{Pr}\left(q=i \Delta+\frac{\Delta}{2}\right), \quad-\infty<i<\infty,
$$

and consider a stochastic signal $y$ with PDF $p_{y}(y)$. If the measurement is quantized, i.e., $q=\mathcal{Q}_{\infty}(y)$, then

$$
p_{i}=\int_{i \Delta}^{(i+1) \Delta} p_{y}(y) d y .
$$

This integral can equivalently be expressed as convolving the signal distribution $p_{y}(y)$ with a uniform distribution

$$
p_{u}(u)= \begin{cases}\frac{1}{\Delta}, & -\frac{\Delta}{2} \leq u \leq \frac{\Delta}{2} \\ 0, & \text { otherwise }\end{cases}
$$


followed by sampling in the regular points $i \Delta+\frac{\Delta}{2}$, [23]. Defining the pulse train $l(q)=\sum_{i=-\infty}^{\infty} \delta\left(q-i \Delta+\frac{\Delta}{2}\right)$, the discrete PDF for $q$ is given as

$$
\begin{aligned}
p_{q}(q) & =l(q)\left(p_{y} \star p_{u}\right)(q) \\
& =\sum_{i=-m}^{m-1} \delta\left(q-i \Delta+\frac{\Delta}{2}\right) \int p_{y}(u) p_{u}(q-u) d u
\end{aligned}
$$

\subsection{Aliasing in the Characteristic Function}

The characteristic function (CF), defined as the Fourier transform (FT) of the PDF, is given as

$$
\Phi_{q}(\varpi)=\mathcal{F}\{p(q)\}=\mathbb{E}\left(\mathrm{e}^{j \varpi q}\right)=\int_{-\infty}^{\infty} \mathrm{e}^{j \varpi q} p(q) d q,
$$

where $\varpi$ denotes the "frequency variable" in the CFdomain. Note that the frequency is sign reversed compared to the usual definition of the FT, but here the CF will be referred to as the FT of the PDF. Hence, with $L(\varpi)=\mathcal{F}\{l(q)\},(11)$ implies that the CF for $q$ is

$$
\begin{aligned}
\Phi_{q}(\varpi) & =L \star\left(\Phi_{y} \Phi_{u}\right)(\varpi) \\
& =\sum_{k=-\infty}^{\infty} \Phi_{y}\left(\varpi+k \frac{2 \pi}{\Delta}\right) \operatorname{sinc}\left(\frac{\Delta\left(\varpi+k \frac{2 \pi}{\Delta}\right)}{2}\right)
\end{aligned}
$$

where $\Phi_{u}(\varpi)=\operatorname{sinc}(\Delta \varpi / 2)=\sin (\Delta \varpi / 2) /(\Delta \varpi / 2)$. For details on characteristic functions, see [9, 19].

From (13), a kind of quantization "aliasing" is introduced, similar to Poisson's summation formula. That is, standard PDFs imply quantization aliasing, which means that high frequencies (fast variations) in the PDF will be interpreted as low frequencies (slow variations). This can be avoided according to QT I if the CF is "band-limited". Such an "anti-alias" condition for quantization is thus

$$
\Phi_{y}(\varpi)=0, \quad|\varpi| \geq \pi / \Delta .
$$

The excellent survey [20] summarizes a range of statistical properties. One key result in this context is the Schuchman's condition [17], which relaxes (14) to that the CF must be zero at regular points $\Phi_{d}(k / \Delta)=0$ for all $k \neq 0$. Note that the Schuchman condition is satisfied for our definition of band-limited noise. Constructive ways to generate band-limited noise are given in Section 4 .

\subsection{Reconstruction of $C F$ and $P D F$}

It follows directly from (13) that the CF for nonquantized measurement can be reconstructed as

$$
\Phi_{y}(\varpi)= \begin{cases}\frac{\Phi_{q}(\varpi)}{\operatorname{sinc}(\Delta \varpi / 2)}, & |\varpi|<\frac{\pi}{\Delta}, \\ 0, & |\varpi| \geq \frac{\pi}{\Delta}\end{cases}
$$

if the anti-alias condition (14) is satisfied, and thus the complete PDF can be constructed.

\section{Generating Band-Limited Noise}

Clearly, standard PDFs, as the Gaussian one, do not satisfy band-limitedness. Note that one does not obtain a band-limited noise by simply truncating $\Phi_{d}(\varpi)$ for $|\varpi|>$ $\pi / \Delta$, since then the PDF will lose its non-negativeness. This section describes some practical methods to obtain band-limited PDFs. In the sequel, only band-limited noise and aliasing in the quantization sense is discussed.

\subsection{Band-Limited Noise}

In Theorem 1, a general class of band-limited PDFs is presented, on which two algorithms will be based.

\section{Theorem 1 Band-limited noise}

A sufficient condition for a PDF to be band-limited in the sense $\Phi_{d}(\varpi)=\mathcal{F}\left\{p_{d}(d)\right\}=0$ for $|\varpi| \geq \pi / \Delta$ is that its $\mathrm{CF}$ can be factored as

$$
\Phi_{d}(\varpi)=\mathcal{H} \star \mathcal{H}(\varpi)=\int \mathcal{H}(s) \mathcal{H}^{*}(\varpi-s) d s
$$

where the complex valued function $\mathcal{H}(\varpi)$ satisfies

$$
\mathcal{H}(\varpi)=0, \text { for }|\varpi|>\frac{\pi}{2 \Delta} \text { and } \int_{-\frac{\pi}{2 \Delta}}^{\frac{\pi}{2 \Delta}}|\mathcal{H}(\varpi)|^{2} d \varpi=2 \pi .
$$

Proof: First, the support of $\mathcal{H} \star \mathcal{H}(\varpi)$ is $[-\pi / \Delta, \pi / \Delta]$, so $\Phi(\varpi)$ is band-limited. Using the well-known Fourier relations $\mathcal{H} \star \mathcal{H}(\varpi) \leftrightarrow|\mathfrak{h}(d)|^{2}$ and Parseval's formula, (17) immediately gives $p_{d}(d)=|\mathfrak{h}(d)|^{2}>0$ and

$$
\int p_{d}(d) \mathrm{d} d=\int|\mathfrak{h}(d)|^{2} \mathrm{~d} d=\frac{1}{2 \pi} \int|\mathcal{H}(\varpi)|^{2} \mathrm{~d} \varpi=1 .
$$

which are the two conditions posed on a PDF. This proves sufficiency.

The perfect anti-alias noise with a flat $\mathrm{CF}$ would have $\mathcal{H} \star \mathcal{H}(\varpi)$ being constant for $|\varpi|<\pi / \Delta$. This would 
imply that $p_{d}(d) \sim \operatorname{sinc}(\pi d / \Delta)$, which is not positive and thus not a valid PDF. This shows that perfect antialias noise does not exist, since the function is not always positive which is in parallel to sampling theory, where the ideal anti-alias low pass filter is not realizable.

\subsection{Construct BL PDFs from a Symmetric PDF}

Theorem 1 indicates a constructive method to define noise PDFs that enable perfect reconstruction after quantization. Take an arbitrary $\mathrm{PDF} p_{d}(d)$. It can be split up as $p_{d}(d)=|\mathfrak{h}(d)|^{2}=\mathfrak{h}(d) \mathfrak{h}^{*}(d)$, yielding $\Phi_{d}(\varpi)=$ $\mathcal{H} \star \mathcal{H}(\varpi)$. This problem is quite similar to spectral analysis and the spectral factorization theorem. One major difference here is the phase ambiguity, $\mathcal{H}(\varpi)$ does not need to be minimum phase.

Assume to start with, that $p_{d}(d)$ is a symmetric PDF. That is, it is an even function. Hence, $\mathfrak{h}(d)$ can be assumed real, and $\mathcal{H}(\varpi)$ becomes a real function as well. This leads to Alg. 1.

\begin{tabular}{l}
\hline Alg. 1 Band-limited approximation of a symmetric PDF. \\
Start with an arbitrary symmetric PDF $p_{d}(d)$. \\
(1) Define $\mathfrak{h}(d)=\sqrt{p(d)}$. \\
(2) Compute $\mathcal{H}(\varpi)=\mathcal{F}\{\mathfrak{h}(d)\}$. \\
(3) Truncate it to $\hat{\mathcal{H}}(\varpi)=\mathcal{H}(\varpi)$ for $|\varpi|<\frac{\pi}{2 \Delta}$. \\
(4) Inverse transform to $\hat{\mathfrak{h}}(d)=\mathcal{F}^{-1}\{\hat{\mathcal{H}}(\varpi)\}$. \\
(5) Normalization constant $c_{\sigma}=\left(\int \hat{\mathfrak{h}}^{2}(d) \mathrm{d} d\right)^{-1}$. \\
(6) Square the result and take $\hat{p}(d)=c_{\sigma} \hat{\mathfrak{h}}^{2}(d)$.
\end{tabular}

\subsection{Construct BL PDFs from Sinc Functions}

Inspired by (15) and Theorem 1 it is clear that Sinc functions can be used to construct PDFs that are perfectly band-limited, with a few finite moments as given in Alg. 2, where the method was independently developed in $[7,11,22]$. The normalization constant and the

$$
\begin{gathered}
\overline{\text { Alg. 2 Band-limited symmetric PDF, }[7,22] .} \\
\text { Take an integer } k \geq 1 \text { and define the PDF as } \\
p_{d}(d)=\frac{1}{c} \operatorname{sinc}^{2 k}(\pi d /(2 k \Delta))=\frac{1}{c} \frac{\sin ^{2 k}\left(\frac{\pi d}{2 k \Delta}\right)}{\left(\frac{\pi d}{2 k \Delta}\right)^{2 k}}, \quad \text { (19a) } \\
\qquad=\int \operatorname{sinc}^{2 k}(\pi d /(2 k \Delta)) \mathrm{d} d .
\end{gathered}
$$

first non-zero finite moments are listed in Table 1.

Example 3 (Band-limited PDFs) First, Alg. 1 is used to approximate the Gaussian standard $\mathrm{PDF} \mathcal{N}\left(0, \sigma^{2}\right)$ with a band-limited one. The result for quantization level
Table 1

Normalization constant and first non-zero moments of the PDF in Alg. 2.

\begin{tabular}{|c|c|c|c|c|}
\hline $\mathbf{k}$ & $\mathbf{c}$ & $\mathbb{E}\left(\mathbf{d}^{\mathbf{2}}\right)$ & $\mathbb{E}\left(\mathbf{d}^{\mathbf{4}}\right)$ & $\mathbb{E}\left(\mathbf{d}^{\mathbf{6}}\right)$ \\
\hline \hline 1 & $2 \Delta$ & $\infty$ & $\infty$ & $\infty$ \\
\hline 2 & $\frac{8}{3} \Delta$ & $\frac{12}{\pi^{2}} \Delta^{2}$ & $\infty$ & $\infty$ \\
\hline 3 & $\frac{33}{10} \Delta$ & $\frac{180}{11 \pi^{2}} \Delta^{2}$ & $\frac{9720}{11 \pi^{4}} \Delta^{4}$ & $\infty$ \\
\hline 4 & $\frac{1208}{315} \Delta$ & $\frac{3360}{151 \pi^{2}} \Delta^{2}$ & $\frac{215040}{151 \pi^{4}} \Delta^{4}$ & $\frac{25804800}{151 \pi^{6}} \Delta^{6}$ \\
\hline
\end{tabular}
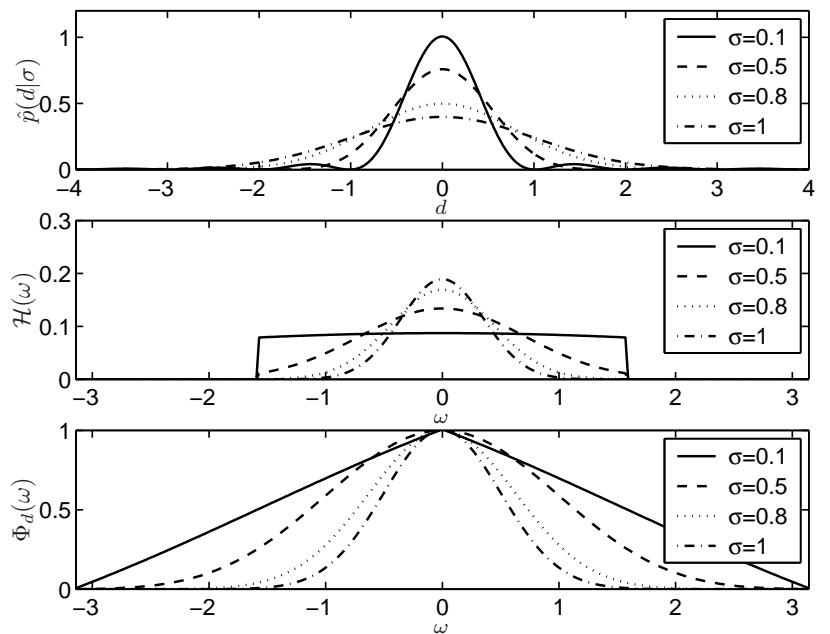

Fig. 2. Illustration of Alg 1: The Gaussian PDF, the spectral factorization of its $\mathrm{CF}, \mathcal{H}(\varpi)$, and the $\mathrm{CF}, \Phi_{d}(\varpi)$, for different standard deviations, $\sigma$, when $\Delta=1$.
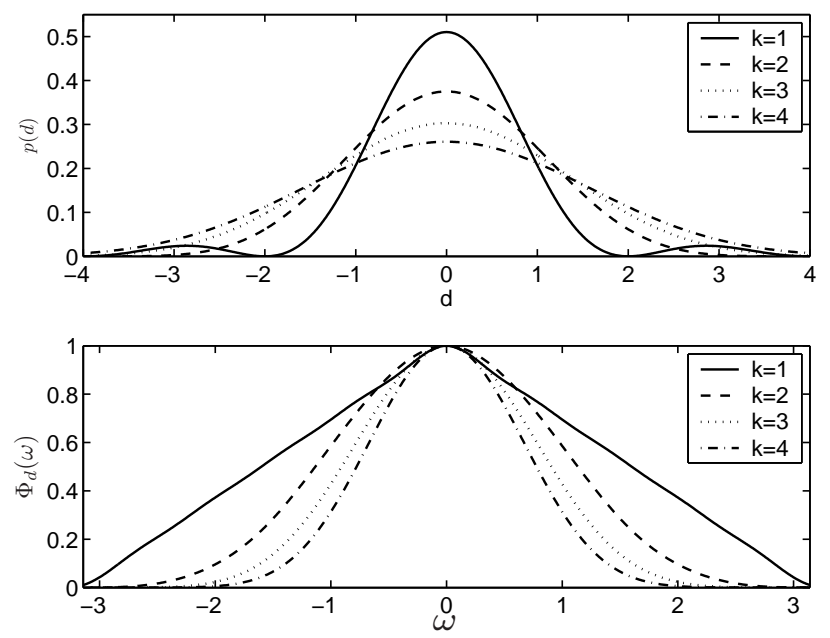

Fig. 3. Illustration of Alg 2: The symmetric BL PDF $p(d)=c \operatorname{sinc}^{2 k}(\pi d /(2 k \Delta))$ and its $\mathrm{CF}$, for $k=1, \ldots, 4$, and $\Delta=1$.

$\Delta=1$ and different standard deviations $\sigma$ is illustrated in Fig. 2. Second, the symmetric PDF $p(d)$ from Alg. 2 for $\sigma=1$ and $\Delta=1$ for $k=1, \ldots, 4$ is given in Fig. 3 . As can be seen, the resulting $\mathrm{PDF} s$ are quite similar for the two algorithms. 


\subsection{Generating Band-limited Dithering Noise}

Alg. 1-2 can be used to generate dithering noise in the following way. Take any uniform random number generator providing random numbers $v_{i} \in[0,1]$. Then, compute the cumulative distribution function $\hat{P}(\cdot)$, and use this to compute $d_{i}=\hat{P}^{-1}\left(v_{i}\right)$, which are then independent samples from $\hat{p}(d)$. Alternatively, the PDF obtained from one of the algorithms can be used directly using accept-reject sampling. See for instance [16] for details. To generate samples from a given $p(x)$ using acceptreject sampling, the assumptions are that there is a proposal density $\bar{p}(x)$ with at least the same support as $p(x)$ and a constant $M$ such that $M \bar{p}(x)>p(x)$ for all $x$. For the PDFs in Alg 2, the tails are quite slowly decaying, and the Cauchy distribution $\bar{p}(x)=1 /\left(\pi\left(1+x^{2}\right)\right)$ is one candidate as proposal distribution. The method is summarized in Alg 3. For small values of $k, M=2$ sat-

Alg. 3 Band-limited noise using accept-reject sampling. The following steps generate samples from the class of distributions in Alg 2 for any $k$ :

(1) Generate a uniform sample $v$, and apply the transformation $x=\tan (\pi(v-0.5))$.

(2) Generate a uniform random value $u$

(3) Accept the proposal sample $x$ if

$$
u<\frac{c_{k} \operatorname{sinc}^{2 k}\left(\frac{\pi x}{2 k}\right)}{M \frac{1}{\pi} \frac{1}{1+x^{2}}} .
$$

The accepted samples can be scaled to $d=x / \Delta$.

isfies the accept-reject condition. The acceptance ratio is close to 0.5 , which means that about one half of the generated samples are accepted.

\section{MLE and CRLB Analysis}

Without quantization, the MLE is defined by (3). Efficient numerical solvers implementing for instance GaussNewton algorithms are routinely used to find the maximum of the likelihood function. For quantized data, there are basically three ways to proceed (see Theorem 2 for notations):

(1) Use the analytical likelihood for quantized data expressed as a discrete PDF $p_{i \mid \theta}(q)$. This is efficiently implemented for a scalar parameter $\theta$ and scalar observation $q$, but in the multi-dimensional case it may lead to computing multi-dimensional integrals and applying an exhaustive search over the quantization levels.

(2) Use the analytical likelihood for quantized data expressed as a continuous function $p_{q \mid \theta}(q(\theta))$. This likelihood can be seen as an analytic continuation of the discrete likelihood above, which enables gradient computations and a numerical search for lo- cal maxima. In analogy with Poisson's summation formula, this likelihood is given by an infinite sum.

(3) Use dithering noise, which enables a rather simple modification of the original likelihood, where standard software can be re-used. The price for this is a slightly increased variance error due to the dithering, but the MLE is as always consistent (asymptotically in the information, the bias and variance decay to zero).

These approaches are investigated and compared in the sequel. The negative effect of the dithering noise will be analyzed in terms of the Cramér Rao lower bound (CRLB).

\subsection{Likelihood Function using Band-Limited Dithering}

The following theorem shows how BL noise using for instance Alg. 1 or 2 can be used to simplify the likelihood.

\section{Theorem 2 (Quantized likelihood function)}

Consider the likelihood function $p_{q \mid \theta}\left(q_{k}\right)$ of the signal model (1), where $q_{k}=\mathcal{Q}_{m}\left(z_{k}(\theta)+d_{k}\right)$ and the dithering noise $d_{k}$ is band-limited and independent of the signal $z_{k}(\theta)$. The unsaturated and saturated likelihood functions are given by

$p_{q \mid \theta}^{\infty}\left(q_{k}\right)=p_{z \mid \theta} \star p_{d} \star p_{u}\left(q_{k}\right)$,

$p_{q \mid \theta}^{m}\left(q_{k}\right)=\left\{\begin{array}{l}\int_{-\infty}^{-(m-1) \Delta} p_{q \mid \theta}^{\infty}\left(q_{k}\right) \mathrm{d} q, \quad q=-m \Delta+\frac{\Delta}{2} \\ p_{q \mid \theta}^{\infty}\left(q_{k}\right), \quad-(m-1) \Delta<q<(m-1) \Delta \\ \int_{(m-1) \Delta}^{\infty} p_{q \mid \theta}^{\infty}\left(q_{k}\right) \mathrm{d} q, \quad q=m \Delta-\frac{\Delta}{2},\end{array}\right.$

respectively, for $q \in\left\{i \Delta-\frac{\Delta}{2}\right\}_{i=-(m-1)}^{m}$.

Proof: The discrete likelihood follows from (8) and (9)

$$
p_{i \mid \theta}(q)=\operatorname{Pr}\left(q=i \Delta-\frac{\Delta}{2} \mid \theta\right)=\int_{i \Delta}^{(i+1) \Delta} p_{z \mid \theta} \star p_{d}(y(\theta)) \mathrm{d} y,
$$

where $p_{z \mid \theta}$ is the original likelihood, $p_{d}$ is the dithering noise PDF and $y(\theta)=z(\theta)+d$. A continuous likelihood that coincides with $p_{i \mid \theta}(q)$ at $q=i \Delta-\Delta / 2$ follows from (11) using the definition of convolution,

$$
p_{q \mid \theta}(q)=\sum_{i=-\infty}^{\infty} \delta\left(q-i \Delta+\frac{\Delta}{2}\right) p_{z \mid \theta} \star p_{d} \star p_{u}(q) .
$$

Here $p_{u}$ the PDF of uniform noise as defined in (10). 
The reconstruction formula (15) corresponds to

$$
\Phi_{q \mid \theta}(\varpi)= \begin{cases}\Phi_{y}(\varpi) \operatorname{sinc}(\Delta \varpi / 2), & |\varpi|<\frac{\pi}{\Delta}, \\ 0, & |\varpi| \geq \frac{\pi}{\Delta},\end{cases}
$$

The sinc function is recognized as the Fourier transform $\Phi_{u}$ of $p_{u}$ in (10). Now, since $\Phi_{y}(\varpi)=\Phi_{q \mid \theta}(\varpi) \Phi_{d}(\varpi)$ and $p_{d}$ is band-limited,

$$
\Phi_{q \mid \theta}(\varpi)=\Phi_{z \mid \theta}(\varpi) \Phi_{d}(\varpi) \Phi_{u}(\varpi)
$$

The inverse Fourier transform now gives (20). Applying saturation to (20) gives (21).

Note that the proof shows that the quantizer can be split into an unsaturated quantizer followed by a saturation as illustrated in Fig. 4. The saturation block has an important role to catch the large amplitude values in the dithering noise.

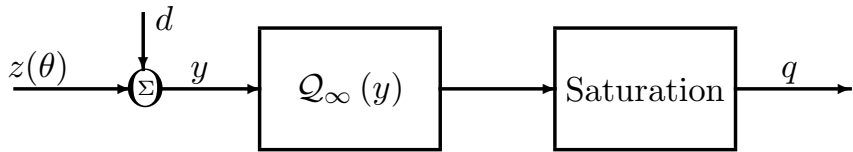

Fig. 4. Saturated quantization seen as unsaturated quantization followed by saturation in series.

The following corollary shows that the situation simplifies when the likelihood function is symmetric. This can be seen as a generalization of [5].

\section{Corollary 1 (MLE for symmetric likelihoods)}

Suppose the likelihood function $p_{y \mid \theta}\left(y_{k}\right)$ is symmetric in $y_{k}$. Then, the MLE for quantized observations using dithering noise from Alg. 1 or 2 can be computed as

$$
\hat{\theta}=\arg \max _{\theta} \prod_{k=1}^{N} p_{y \mid \theta}\left(q_{k}\right) .
$$

Proof: The dithering noises in Alg. 1 and 2 are both symmetric. Thus, the likelihood functions in (20) and (21) will also be symmetric, and they get the same global maximum as the unquantized likelihood function. That is, asymmetric likelihood functions as in Example 2 are particularly challenging.

\subsection{Cramér-Rao Lower Bound (CRLB)}

Consider initially the fundamental problem of estimating the mean $\theta$ from the quantized measurements $q=$ $\mathcal{Q}_{m}(\theta+e+d)$. For an unbiased estimator, the expected mean equals the true parameter, $\mathbb{E}(\hat{\theta})=\theta$. The CRLB, $[4,12,14]$, is then given by

$$
\operatorname{Cov}(\theta-\hat{\theta})=\mathbb{E}\left((\theta-\hat{\theta})(\theta-\hat{\theta})^{T}\right) \succeq J^{-1}(\theta),
$$

Table 2

The FIM of the PDF in Alg. 2.

\begin{tabular}{|l|r|r|r|r|}
\hline $\mathbf{k}$ & 1 & 2 & 3 & 4 \\
\hline \hline $\mathbf{J}^{\mathbf{1 / 2}}(\mathbf{d})$ & $0.56 \Delta$ & $1.00 \Delta$ & $1.28 \Delta$ & $1.50 \Delta$ \\
\hline
\end{tabular}

where $J(\theta)$ denotes the Fisher information matrix (FIM). Details on how to compute the CRLB is given in $[8,11,13]$. The purpose here is to point out the implications of using band-limited dithering noises. We summarize the CRLB results in terms of a simple yet characteristic example, where the mean of a signal is estimated.

In Fig. 5 the Fisher information $J_{m}(\theta)$ is illustrated without dithering for the mean estimation problem, see [8] for more details, by plotting the lower bound $J_{m}^{-1 / 2}(\theta)$ for different quantization levels $\Delta=2 / m$ with $e \in$ $\mathcal{N}\left(0,0.1^{2}\right)$. Note that:

- The CRLB is parameter dependent. For some values of the true $\theta$ (close to the quantization steps), accurate estimation is expected.

- Asymptotically as $\Delta \rightarrow 0$, the quantization effects disappear. In this example, $J_{100}^{-1 / 2}(\theta) \approx \sigma$.

- In practice, quantization is often approximated with the AUN assumption. This corresponds to the contribution $\frac{\Delta^{2}}{12}$, which is a quite coarse approximation. For this example, the AUN assumption corresponds to a smoothing of each curve. The transformed Fisher information for the sum of Gaussian and uniform noise is given by

$$
J_{m}^{-1 / 2}(\theta)=\sqrt{\sigma^{2}+\Delta^{2} / 12}=\sqrt{\sigma^{2}+1 /\left(3 m^{2}\right)} .
$$

The latter expression applies to the notation in Fig. 5 . For $m=4, J_{m}^{-1 / 2}(\theta) \approx 0.51$, which is strictly larger than the actual bound.

- With band-limited dithering, the CRLB becomes $J_{m}^{-1 / 2}(\theta) \approx \sqrt{\sigma^{2}+\frac{\Delta^{2}}{12}+F I M(d)}$, where again $\frac{\Delta^{2}}{12}=$ $1 /\left(3 m^{2}\right)$ applies to the case in Fig. 5. The dithering noise implies that quantization can be regarded as AUN in MLE.

- The CRLB contribution $F I M(d)$ is usually in the same order as the AUN, see Table 2. Further, the quantization step $\Delta$ must be at least as large as the measurement noise to make quantization a problem. That means that the total FIM increases a factor of two approximately due to dithering.

We next extend the mean estimation problem to the general parameter estimation problem in Theorem 3.

Theorem 3 Consider the signal model

$$
q_{k}(\theta)=\mathcal{Q}_{m}\left(z_{k}(\theta)+d_{k}\right) .
$$

The Fisher information for a set of quantized measurements is given by $J(\theta)=\sum_{k=1}^{N} H_{k}^{T} J\left(z_{k}(\theta)\right) H_{k}$, where 


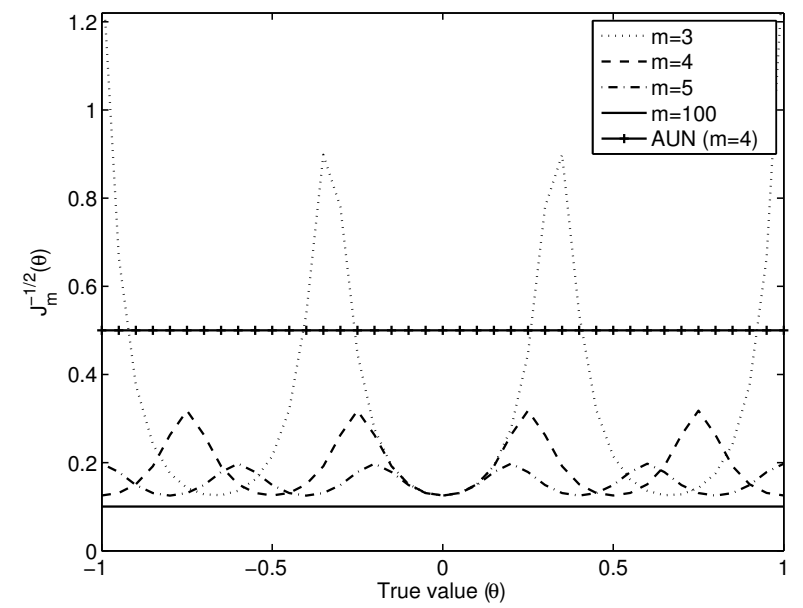

Fig. 5. Fisher information for $q=\mathcal{Q}_{m}(\theta+e)$ without dithering, where the lower bound $J_{m}^{-1 / 2}(\theta)$ is plotted as a function of $\theta$ for different quantization levels $\Delta=2 / \mathrm{m}$. For AUN dithering note that $\sqrt{\Delta^{2} / 12}=2 / m=0.5$ for $m=4$.

$H_{k}=\frac{d}{d \theta} z_{k}(\theta)$ and $J^{-1}\left(z_{k}(\theta)\right)=\sigma^{2}+\frac{\Delta^{2}}{12}+F I M(d)$.

Proof: Follows from the chain rule and the additivity of information.

This result is useful to extend known results on CRLB for unquantized signals to the case of quantization.

\section{Conclusions}

The advantage of dithering as a pre-processing step before quantization is well-known in practice. One of the few known theoretical properties in statistical signal processing terms is that all moments up to order $r$ can be computed analytically if $r$ uniformly distributed random numbers are added to each signal sample. We have studied the strictly stronger condition of band-limitedness of the dithering noise. It is defined by restricting the Fourier transform of the dithering noise PDF to have compact support. Two constructive methods were provided to generate samples from such dithering noise, supported by a concrete algorithm based on accept-reject sampling. The advantage is that the amplitude distribution of the signal can be recovered exactly, not only all of its moments. One of the most interesting implications for signal processing, is the possibility to compute the exact likelihood for quantized and dithered signals. In this way, the statistically efficient ML estimator can be applied, to asymptotically avoid the bias that all other dithering noises would suffer from. The implication for system identification is that standard numerical search algorithms can be applied for parameter estimation, with a minor modification. This modification includes a spatial smoothing of the non-dithered and nonquantized likelihood function.

\section{References}

[1] J.C. Agüero, G.C. Goodwin, and J.I. Yuz. System identification using quantized data. In IEEE Conference on Decision and Control, volume 46, 2007.
[2] T.C. Aysal, M.J. Coates, and M.G. Rabbat. Distributed average consensus with dithered quantization. IEEE Transactions on Signal Processing, 56(10):4905-4918, 2008.

[3] L. Balogh, I. Kollar, and A. Sarhegyi. Maximum likelihood estimation of ADC parameters. In IMTC 2010, pages 24-29, Austin, Texas, U.S.A, June 2010.

[4] H. Cramér. Mathematical Methods of Statistics. Princeton, NJ: Princeton University Press, 1946.

[5] O. Dabeer and A. Karnik. Signal parameter estimation using 1-bit dithered quantization. IEEE Trans. Information Theory, 52(12):5389-5405, 2006.

[6] O. Dabeer and E. Masry. Multivariate signal parameter estimation under dependent noise from 1-bit dithered quantized data. IEEE Trans. Information Theory, 54(4):1637-1654, 2008.

[7] F. Gustafsson and R. Karlsson. Statistical results for system identification based on quantized observations. In Proc. 15th IFAC Symposium on System Identification, 2009.

[8] F. Gustafsson and R. Karlsson. Statistical results for system identification based on quantized observations. Automatica, (45):2794-2801, December 2009.

[9] A. Gut. An Intermediate Course in Probability. Springer-Verlag, 1995.

[10] G.W. Wornell H.C. Papadopoulos and A.V. Oppenheim. Sequential signal encoding from noisy measurements using quantizers with dynamic bias control. IEEE Trans. Information Theory, 47(3):978-1002, 2001.

[11] R. Karlsson. Particle Filtering for Positioning and Tracking Applications. $\mathrm{PhD}$ thesis, Linköping University, Linköping, Sweden, 2005. Dissertations No. 924.

[12] S.M. Kay. Fundamentals of signal processing - estimation theory. Prentice Hall, 1993.

[13] R.L Landes. Statistical Methods for Application to Calibration Problems. PhD thesis, Iowa State University, 2005.

[14] E. L. Lehmann. Theory of Point Estimation. John Wiley and Sons, 1983.

[15] A. Oppenheim and R. Schafer. Digital Signal Processing. Prentice-Hall, 1975.

[16] C. P. Robert and G. Casella. Monte Carlo Statistical Methods. Springer-Verlag, 1999.

[17] L. Schuchman. Dither signals and their effect on quantization noise. IEEE Transaction on Communication Technology, 12:162-165, 1964.

[18] A.B. Sripad and D.L. Snyder. A necessary and sufficient condition for quantization errors to be uniform and white. IEEE Transactions on Signal Processing, 25:442-448, 1977.

[19] A. Stuart and J. K. Ord. Kendall's Advanced Theory of Statistics, volume 1. London: Edward Arnold, NewYork Wiley, 6 edition, 1994.

[20] R.A. Wannamaker, S.P. Lipshitz, J. Vanderkooy, and J.N. Wright. A theory for nonsubtractive dither. IEEE Transactions on Signal Processing, 48(2):499-516, 2000.

[21] B. Widrow. A study of rough amplitude quantization by means of Nyquist sampling theory. IRE Transactions on Circuit Theory, pages 266-276, December 1956.

[22] B. Widrow and I. Kollar. Quantization Noise: Roundoff Error in Digital Computation, Signal Processing, Control, and Communications. Cambridge University Press, 2008.

[23] B. Widrow, I. Kollar, and M-C. Liu. Statistical theory of quantization. IEEE Transactions on Signal Processing, pages 353-361, April 1996. 